\title{
The morphological and ecological variation of Arctostaphylos (Ericaceae) fruit: a link between plant ecology and animal foraging behavior
}

\author{
Rebecca Crowe ${ }^{1}$ and V. Parker ${ }^{2}$ \\ ${ }^{1}$ University of California Irvine \\ ${ }^{2}$ San Francisco State University College of Science and Engineering
}

February 27, 2022

\begin{abstract}
Persistent soil seed banks are characteristic of Arctostaphylos (Ericaceae) species in the Mediterranean-climate California Floristic Province. While most species are obligate seeders, regeneration of stands of all Arctostaphylos species ultimately depends on post-fire seedling recruitment. Arctostaphylos seed banks are created, in large part, by scatter-hoarding rodents. Variation in fruit morphology, therefore, is expected to impact the Arctostaphylos-rodent interaction. Seeds produce sufficient rewards (nutritious mature embryo) to entice rodents to disperse and ultimately bury seeds in the soil. Hard seed coats increase the time required to extract the embryo, encouraging rodents to choose storage over immediate predation, and endocarps are frequently empty. We assessed the variation of fruit endocarp fusion and seed viability among species of Arctostaphylos. Factors such as latitude, elevation, life history, ploidy, and phylogenetic position were also analyzed. Generalized mixed effects models were used to determine the factors contributing to variation in fruit endocarp fusion and viability. Our results indicate that fruit volume and shape are the most important variables affecting endocarp fusion and seed viability. Additionally, other potential influences only show a weak correlation and are not predicted to significantly impact fruit endocarp fusion or viability. These findings provide insight into evolved strategies used by plants to increase reproductive success via scatter-hoarding rodents. Our study benefits the conservation and restoration of Arctostaphylos stands by emphasizing the importance of animal-mediated dispersal and providing estimates of seed viability for different species. With the anticipated effects of climate change, such as departures from historic fire regimes, the preservation of the relationship between plants and animal foragers is crucial for the continued survival of Arctostaphylos and California's evergreen chaparral.
\end{abstract}

\section{Title}

The morphological and ecological variation of Arctostaphylos(Ericaceae) fruit: a link between plant ecology and animal foraging behavior

\section{Authors}

Rebecca E. Crowe ${ }^{1,2,3}$

V. Thomas Parker ${ }^{1}$

Crowe ORCID: 0000-0003-4133-8692

Parker ORCID: 0000-0002-6149-7140

${ }^{1}$ San Francisco State University, San Francisco, CA 94132 
${ }^{2}$ Current address: UCI Herbarium, Ecology \& Evolutionary Biology, University of California, Irvine, Irvine, CA $92697-2525$

${ }^{3}$ Author for correspondence: rebeccaecrowe@gmail.com

parker@sfsu.edu

\begin{abstract}
Persistent soil seed banks are characteristic of Arctostaphylos(Ericaceae) species in the Mediterranean-climate California Floristic Province. While most species are obligate seeders, regeneration of stands of all Arctostaphylos species ultimately depends on post-fire seedling recruitment. Arctostaphylos seed banks are created, in large part, by scatter-hoarding rodents. Variation in fruit morphology, therefore, is expected to impact theArctostaphylos -rodent interaction. Seeds produce sufficient rewards (nutritious mature embryo) to entice rodents to disperse and ultimately bury seeds in the soil. Hard seed coats increase the time required to extract the embryo, encouraging rodents to choose storage over immediate predation, and endocarps are frequently empty. We assessed the variation of fruit endocarp fusion and seed viability among species of Arctostaphylos . Factors such as latitude, elevation, life history, ploidy, and phylogenetic position were also analyzed. Generalized mixed effects models were used to determine the factors contributing to variation in fruit endocarp fusion and viability. Our results indicate that fruit volume and shape are the most important variables affecting endocarp fusion and seed viability. Additionally, other potential influences only show a weak correlation and are not predicted to significantly impact fruit endocarp fusion or viability. These findings provide insight into evolved strategies used by plants to increase reproductive success via scatter-hoarding rodents. Our study benefits the conservation and restoration of Arctostaphylosstands by emphasizing the importance of animal-mediated dispersal and providing estimates of seed viability for different species. With the anticipated effects of climate change, such as departures from historic fire regimes, the preservation of the relationship between plants and animal foragers is crucial for the continued survival of Arctostaphylos and California's evergreen chaparral.
\end{abstract}

\title{
Keywords:
}

Fruit morphology; scatter-hoarding rodents; fire regime; dispersal; seed viability; generalized mixed effects model

\section{Introduction and background}

Propagule dispersal, the movement of seeds and fruit away from the parent plant by biotic or abiotic agents, is a significant part of a plant's life history and critical to the dynamics of plant communities (Venable and Brown 1988, Wang and Smith 2002, Levine and Murrell 2003). Plants are influenced by dispersal at the individual level (selection on reproductive traits such as seed morphology), population level (gene flow), and community level (community assembly) (Lichti et al.2015). The interaction between plants and their dispersal vectors influences seed and fruit morphology and chemistry, whether the vectors are abiotic dispersal agents like wind, water, or gravity or diffuse biotic dispersal agents like animals (Tiffney 2004, Rubio de Casaset al. 2015). Scatter-hoarding represents a particular type of dispersal in which animals collect, bury, manage, and consume seed in numerous small caches in the soil over long time periods (Vander Wall 2001). Scatter-hoarding rodents are both consumers and dispersers of seed and fruit and variation in propagule traits may influence the balance between those processes (Wang et al. 2012). Fruit and seed traits provide important insight into the discrete traits that are likely under selection by dispersal agents and how the traits in turn may be influencing or reinforcing animal behavior. We focus here on morphological variation in fruit and seed among species in the plant genus Arctostaphylos (Ericaceae) whose fruit and seed are buried by scatter-hoarding rodents (Parker 2015a).

Fire-stimulated, persistent soil seeds banks are characteristic of Arctostaphylos species in the Mediterraneanclimate California Floristic Province (Keeley 1977, Parker and Kelly 1989). While persistent soil seed bank 
formation is in part dependent upon seed size, because smaller seeds typically generate larger seed banks as they have better architecture for abiotic burial mechanisms (Thompson et al. 1993), the fruit and seeds of Arctostaphylos species are generally too large and round to accumulate in the soil by these processes alone, yet have relatively dense soil seed banks (Parker and Kelly 1989, Parker 2015a, Parker and Ingalls 2022). Scatter-hoarding rodents turn out to be effective dispersers of Arctostaphylos by creating multiseeded caches that are shallowly buried in soil and litter (Moore and Vander Wall 2015, Parker 2015b). Almost allArctostaphylos seedlings emerge from rodent caches following a high intensity fire, and 40-60\% of seedlings were ascribed to rodent caches in medium intensity fires (Parker 2015b). Caching of seeds by rodents increases the chances of Arctostaphylos seed survival in soil seed banks during fires even at depths with an otherwise killing heat pulse (Peterson and Parker 2016).

Arctostaphylos has unusually large seed banks given seed size, especially when compared to a similar California genus, Ceanothus(Keeley 1977, Parker and Kelly 1989, Kelly and Parker 1990, Parker 2015a). Annual seed production for both species can vary widely year to year and is influenced by seasonal and annual climate fluctuations due to rainfall and to a smaller extent temperature (Keeley 1977). In favorable years seeds produced exceeds the number of seeds in soil seed bank indicating that many seeds are lost from chaparral stands due to pre- and post-dispersal predation by animals and insects, dispersal by animals, erosion, and decay (Keeley and Hays 1976, Kelly and Parker 1990, O'Neil and Parker 2005). Differences in seed bank size between Ceanothus and Arctostaphylos are due to the behavior difference of primary seed predators; birds and rodents consumeCeanothus seed in contrast with only carnivorous mammals and rodent scatterhoarders that consume and disperse Arctostaphylosfruit and seed (Warzecha and Parker 2014, Moore and Vander Wall 2015, Parker 2015a, 2015b). Considerable post-dispersal seed predation occurs in both genera (Parker 2015a) although, considering seed predators and dispersers, seed size is often a predictor of whether immediate predation occurs or not (Jansen et al. 2002, Muñoz and Bonal 2008, Vander Wall 2010, Wang et al. 2012). The smaller size ofCeanothus seeds versus the larger size of Arctostaphylosseeds may reflect that pattern (Warzecha and Parker 2014, Parker 2015a).

Fruit traits vary in critical dimensions with respects to scatter-hoarding rodents. First, morphology of Arctostaphylosfruit varies in shape and size, with Arctostaphylos producing drupes that range between spheric to vertically depressed-spheric in shape (Figure 1). The drupes also range in size: A. myrtifolia, A. nissenana, $A$. nummularia and $A$. sensitiva have the smallest drupes that are around 3-4 millimeters wide, while the largest fruited species are A. gabilanensis, A. glauca, A. hooveri, A. mewukka, A. refugioensis, and $A$. viridissimawith fruit 10-15 mm wide (Parker et al. 2012). The fruit are composed of three layers. The outermost layer, the exocarp, is dry and thin and ranges in color, glands, and hairs; immature fruit are green or white and ripen to various shades of red and brown. The mesocarp is also dry, and the composition and thickness vary among species from thick and mealy to absent. The third layer is composed of a thickened, stony endocarp surrounding the embryo and associated endosperm. The collective unit of the hardened endocarp, embryo, and endosperm resemble small nuts and are called nutlets. Nutlet traits create a second level of diversity for rodents. Nutlets may be coalesced together into multi-seeded pieces by the fusion of adjacent endocarps and range from no fusion to all endocarp surfaces fused, creating different sizes of potential rewards, among species and even from a single fruit. For example, A. andersonii and A. canescens often have distinct, unfused nutlets, $A$. glandulosa and $A$. patula fruit have some nutlets fused and some distinct, while A. glauca and A. gabilanensis nutlets are fused into a single sphere (Parker et al. 2009). Not all ovules develop into mature seeds, however, creating a third level of diversity for rodent assessment, and mature fruit often contain a mix of empty and seed-filled locules.

Animals select seeds based on the amount of energy and nutrients gained and assess seeds by examining seed traits such as size, composition (total energy, protein, lipids, carbohydrates, indigestibles), secondary metabolites, dormancy, handling costs, and infestation by insects (Lichti et al. 2015). Whether scatter-hoarding animals directly assess seed viability is unknown, but seeds that are cached by rodents are predominantly viable (Jansen et al. 2004) and rodents notice and reject insect infested seeds preferring intact and viable seeds (Perea et al. 2012). Smaller seeds are more likely to experience predation soon after they are discovered, while larger seeds are more often collected and dispersed (Jansen et al. 2002, Muñoz and Bonal 2008, Vander 
Wall 2010, Wang et al. 2012).

Thus, fruit morphology clearly influences the caching behavior of scatter-hoarding rodents. With respect to Arctostaphylos species, rodents will cache whole fruit (Parker 2015b) or nutlets separated from fruit, including endocarps found in coyote or bear scat (Moore and Vander Wall 2015). Because rodents assess and respond to the balance between constraints and rewards, we investigated potential ways rodent behavior may be manipulated in Arctostaphylos. Our overall objective in this study was to determine if fruit variation inArctostaphylos may be an influence on seed bank dynamics associated with scatter-hoarding. Here we examined fruit: 1) for differences in seed fusion among plants and species; 2) to determine the extent to which fusion of seed is influenced by morphology, environmental factors, life history characteristics, and species identity; 3) for differences in fruit viability among species; and 4) to determine the extent to which fruit viability is influenced by morphology, environmental factors, life history characteristics, and species identity.

\section{Materials and methods}

\section{Data Collection}

Arctostaphylos fruit were collected from chaparral and mixed evergreen forests throughout California during the summers and falls of 2013-2016. Opportunistic collections were made from samples from previous studies and these fruit were collected from Northern and Central California sites during 1988-2013. The collections were stratified by fruit size, species life history, species phylogenetic position (clade), and species ploidy so that as many different species as feasible were sampled per combination of factors (Table 1).

Fruit and, when feasible, vouchers were collected from 39Arctostaphylos species (Figure 2) with permission under the permits listed in Appendix 1 . Voucher specimens were deposited at the H.D. Thiers Herbarium (SFSU) with duplicates at the UC Irvine Herbarium (IRVC). Herbarium data is available through the Consortium of California Herbarium (Consortium of California Herbaria (CCH) 2018). Specimens were identified using the Flora of North America, The Jepson Manual, and Field Guide to Manzanitas (Parker et al. 2009, 2012, Kauffmann et al. 2015). A minimum of 33 ripe, undamaged fruit were sampled from each site, and from several individual plants when possible (Table 2). Fruit was brought into the lab at San Francisco State University for measurement of fruit morphologic characters.

We counted the number of viable seeds and fused nutlets per fruit to quantify the extent of variation between individual plants and between species and to identify potential drivers of this variation. For each fruit we counted the total number of nutlets and the number of fused nutlets. We examined each endocarp segment or conglomerate of fused endocarp segments to determine the number of apparent seed chambers, which are the ovary locules (Figure 1). Seed chambers were interpreted as discreet segments delineated either by complete separation or by indentations on surface caused by the fusion of endocarp pieces. The number of seed chambers were confirmed by radially cutting fused endocarp segments in half with a jeweler's hacksaw. We counted the total number of endocarp segments and number of endocarp segments with at least one radial surface fused for each fruit and used this count to derive $\%$ fusion (\# fused chambers/ \# total chambers) for each fruit. Fruit viability was measured as the number of full (viable seeds) seeds to the \# of total seed chambers in one fruit for a subset of 15 Arctostaphylos taxa. Ten of those species had fruit that was collected from more than one plant, while the remaining five species were bulk collections made for previous studies.

Propagule size will vary not only with fruit size, but also with the degree of endocarp fusion, in that fruit with some free nutlets will functionally become multiple smaller propagules over time, once the exocarp and mesocarp are removed due to decay or by animals. Processes that influence fruit size may in turn influence nutlet fusion inArctostaphylos. Previous fruit size studies explored the drivers of fruit size differences across local to global spatial scales. These studies indicated that elevation, latitude, and "droughtiness" influence seed size (Baker 1972, Moles et al. 2007, Simpson et al.2017). Potential environmental drivers of variability in endocarp fusion include elevation, average yearly precipitation, average yearly maximum temperature, climate water deficit, latitude, and longitude. These data were extracted from the PRISM 30-year normals 
baseline dataset that describes the average monthly and annual conditions from 1981-2010, and the Climatic Water Deficit dataset, which is an estimate of drought stress on soils and plants (Table 3) (PRISM Climate Group n.d., Flint and Flint 2014).

In addition to environmental variables, species and fruit-level traits were also measured. The life history and ploidy level of species may potentially influence nutlet fusion and fruit viability. Differences in fruit viability and percent seed set between sprouters and non-sprouters and ploidy level has been documented (Keeley and Keeley 1977, Kelly and Parker 1991). Life history, phylogenetic position (based on Wahlert et al. 2009), and ploidy level of each taxon were recorded as categorical variables. Each categorical variable had two levels; "ns" for non-sprouting or "s" for sprouting species, "large" or "small" clade, and "4n" or "2n" for ploidy level.

We measured six morphological traits of potential ecological importance for each fruit. Fruit height and width were measured, in millimeters, with digital calipers (Mitutoyo Digimatic CD-6"BS); height was measured at the tallest point of the fruit, between the peduncle and the flower end, and width was measured at the widest place along the fruit equator. Fruit shape was calculated as the ratio of fruit height to width and corresponds to the following conventional 3-d shapes: obloid/ ellipsoid (2:1), widely ellipsoid (6:5), globose (1), depressed globose (3:4), oblate (1:2) (Simpson 2010). Fruit volume was calculated as the volume of an oblate spheroid (Simpson 2009). Pulp, the mealy mesocarp layer of fruit present in some Arctostaphylos species, was assessed and assigned a category; thick sheet, scant, absent, powdery, mealy/powdery, mealy, appressed sheet. Surface pubescence and color were visually assessed and categorized. Morphological fruit traits are summarized inTable 4 .

Data Analysis

All analyses were conducted using the $\mathrm{R}$ statistical software ( $\mathrm{R}$ Core Team 2018). We first explored the data with plots to detect outliers, homogeneous variance of independent variables, normality of independent variables, collinearity among covariates, too many zeros, and independent y variables (Zuur et al. 2010). Data exploration confirmed a non-normal distribution of data, non-independent observations, and heterogeneous variance indicating the need to use GLMMs (generalized linear mixed effects models). We used GLMMs to model relationships between fruit endocarp fusion, fruit viability, environmental, morphological, and life history parameters. Response variables were fruit endocarp fusion and fruit viability, which are proportional and include zeros and ones, and are best fit with a binomial distribution and logit-link. Continuous independent variables were centered, mean subtracted from each observation, and scaled. Centering removes the correlation between slope and intercepts and makes the treatment main effects meaningful, i.e. independent of the slopes (Schielzeth 2010).

After "hard thinking" we fit a global model for fruit endocarp fusion using the "lme4" package, determined the structure of the random effects, and determined the structure of the fixed effects (Burnhamet al. 2011, Bates et al. 2015). We established the structure of the random effects part of the model using BIC (Bayesian Information Criterion) to rank several models with different combinations of random effect terms, and the best model was: individual plant (momcode) nested in species with an observation level random effect (Ucode). Many zeros were detected in data exploration, so we included an observation level random effect (OLRE) to account for overdispersion. We checked for overdispersion and the OLRE seemed to adequately address overdispersion $($ chisq $=616$, residual $\mathrm{df}=1712)$.

To determine the best structure for the fixed effects we used the dredge function in the package "mumin" to fit, using the global model, all possible combinations of variables excluding correlated variables and including ecologically sensible two-way interactions. The random effects of species identity, maternal plant, and unique identifier were held constant for all models. We compared the models using BIC and the best model has the lowest BIC.

Eight models were within 7 BIC and the best model included only fruit volume and fruit shape. The next best model was 4.06 BIC higher than the best model, which differed by including the interaction of fruit volume: shape. In other words, the model with fruit fusion and shape was 7.65 times more likely than the next model to be true (Table 5). While models up to 7 BIC different may be considered plausible (Burnhamet 
al. 2011) we did not pursue model averaging because we were also interested in the variation between species and plants (random effects), which are not accounted for in model averaging (Bolkeret al. 2009).

We utilized the same model fit and selection protocol for the fruit viability data set. We found that the best model included plant nested in species as random effects and fruit volume, fruit shape, and the interaction of fruit volume and shape as fixed effects (Table 6). The next best model was 2.70 BIC higher than the best model, which differed by including life history. The best model was 3.85 times more likely than the next best model to be true.

We assessed the fit of the best models for fruit fusion and viability by checking for unexplained patterns in the mean and variance with plots of scaled residuals vs. fitted values and input variables using the DHARMa package in $\mathrm{R}$ and other base $\mathrm{R}$ plots (Hartig 2019).

\section{Results}

\section{Fruit fusion}

We found a range of endocarp fusion among species. For example, no endocarp fusion was observed in fruit of $A$. nortensis, and all endocarps were fused in fruit of A. glauca, A. klamathensis, A. rainbowensis, and A. refugioensis (Table 4) .

As fruit volume increased, the proportion of fused endocarps per fruit likewise increased. A one standard deviation (since we centered and scaled) increase in fruit volume was associated with a 0.546 unit increase in the expected $\log$ odds of percent fusion ( $\mathrm{se}=0.107$, Profile CI: $0.334-0.767$ ), or a predicted probability of $63 \%$, when shape was held at an average value (Table 7, Figure $3 \& 4$ ). Fruit shape, the ratio of height to width, had a positive effect on percent endocarp fusion; a one standard deviation increase in shape (fruit become more elongate) was associated with a 0.526 unit increase in the expected log odds of percent endocarp fusion ( $\mathrm{se}=0.069$, Profile $\mathrm{CI}=0.389-0.669)$, when fruit volume was held at an average value, or a predicted probability of $62.8 \%$ (Figure $3 \& 4$ ) .

More variation in proportion of endocarp fusion was found among species (variance $=3.989, \mathrm{SD}=1.997$ ) than individual fruit (variance $=1.132, \mathrm{SD}=1.064$ ) or plants (variance $=0.626, \mathrm{SD}=0.791$ ) (Table 7, Figure 5). The among-species random effect standard deviation (in log-odds of seed fusion) is comparable to the magnitude of the fixed effects and is greater than the largest single fixed effect. For species level deviations from the intercept, A. nortensis was the most negatively different, and $A$. refugioensis the most positively different (Figure 5) .

Fruit viability

We found a range of seed viability among species; the lowest around $10.27 \%$ of the seeds in each fruit were viable in A. nevadensissubsp. knightii and the highest around $69.92 \%$ of $A$. stanfordiana seeds were viable (Table 8) .

The estimated effect (in log-odds scale) of fruit volume on percent seed viability per fruit was 0.638 (se= 0.105, Profile CI: $0.435-0.848$, Table 9, Figure 6 ), or a predicted probability of $65.4 \%$ (Figure 7) . The estimated effect of the interaction of fruit volume and shape (height:width) on percent seed viability was -0.240 ( $\mathrm{se}=0.063$, Profile CI: $-0.365,-0.116$, Table 9) . The interaction plot shows predicted probabilities of viable seeds in a fruit; the effect of fruit volume on viability was different at the highest and lowest values of fruit shape (ratio of height to width); for fruit with small shape ratios, volume acts to increase viability and for fruit with large shape ratios, larger fruit have lower viability(Figure 8). There was little evidence that shape (height to width) alone affects viable seed because the confidence interval includes zero (Est. = 0.137 , Profile $\mathrm{CI}=0.000-0.277$, Table 9 ).

Variability in viability was similar among plants (variance $=0.579, \mathrm{SD}=0.761$ ) and among species (variance $=0.581, \mathrm{SD}=0.762$, Table 9 ). The among-species standard deviation was 0.762 , which is similar in magnitude to the effect of fruit volume, 0.638 . The random effects plots show the group level deviations from 
the fixed intercept, which illustrates how much a particular plant (momcode:species) or species intercept is shifted towards or away from the fixed intercept (Figure 9 ). For species level deviations from the intercept, $A$. manzanita subsp. elegans (mane) was the most different, as were A. patula (patu), and $A$. montana subsp.montana (monm).

\section{Discussion}

Arctostaphylos species are characterized by persistent soil seed banks mainly created by scatter-hoarding rodents. Interactions between scatter-hoarding rodents and Arctostaphylos should have selected traits of fruit to manipulate the behavior of seed predators, thus permitting the sustainability of their seed banks. We found that variation in fruit morphology supports a model of historical selection for long-term seed survival in the soil in the context of scatter-hoarding interactions. Arctostaphylos fruit traits can influence animal behavior by producing fruit that is more likely to be buried and less likely to be found again after burial by imperfect foragers (Vander Wall 2010). Fruit traits that influence animal behavior are size, nutritive content, hard seed coat, masting, and lack of smell (Vander Wall 2010). Our findings demonstrate that underlying patterns of both fruit endocarp fusion and percent seed viability are best explained by variation in fruit volume and shape including extensive variation between species, plants, and individual fruit. The thick, hardened endocarp offers some protection from predation by increasing handling time, the time it takes to extract the nutritive seed (Vander Wall 2010). Post-burial, endocarp fusion increases the perceived size and is a direct indication of the potential reward available to rodents, while variation in percent viability also increases handling time and a giving-up behavior (Charnov 1976, Brown 1988). The high handling time influences the rodent's assessment of the seed by shifting behavior from eating immediately to saving for later in a cache (Lichti et al. 2015). These results can explain the variation in seed bank densities among these species (Parker and Ingalls, in press ). Because no differences were found among species by phylogenetic clades or life history, we presume these are originally historic patterns. This suggests that endocarp fusion and seed viability patterns associated with scatter-hoarding arose ancestrally within Arctostaphylos, prior to the obligate seeding life history, and are adaptive traits that ensures reproductive success by manipulating scatter-hoarding rodent behavior (Parker 2015a, 2015b).

We expected environmental and life history variables to have an effect on the proportion of fused endocarps and viability per fruit but they were not present in the best models (Table 6, Table 7) and correlation plots do not suggests strong patterns between these independent and dependent variables (available on request). Previous studies demonstrated that temperature, elevation, precipitation, and latitude can affect fruit and seed mass, but these variables in our study do not drive differences in the degree of endocarp fusion and seed viability (Baker 1972, Moles et al. 2007, Simpson et al.2017). We also anticipated a relationship between proportion of fused endocarp and fruit viability, but our data indicates that there is no clear pattern among all the species as a group.

These patterns of fusion and seed viability do, however, reflect seed density variation in persistent seed banks of these species. Fruit volume and fruit shape were the most important variables affecting fruit fusion (Table 5). The effect on viability by volume, however, is different when examined for different types of fruit shape (Figure 8). Percent seed viability per fruit is higher with increasing fruit volume for oblate or depressedglobose shaped fruit, while viability declines with increasing fruit volume for the obloid/ellipsoid, roughly globose shaped fruit. Large, globose fruit tend to have high proportions of endocarp fusion, where all seeds are coalesced into a single structure, and may provide consistent perceived reward sizes for scatter-hoarding rodents and therefore increase their perceived value. Because scatter-hoarding rodents may preferentially disperse larger fruit, the plants may have been selected to not invest as many resources in producing viable seeds per fruit, as the high level of fusion found in large fruit ensures that the entire fruit is dispersed and the lower number of viable seeds increases the chance for an eventual giving-up behavior (Charnov 1976, Brown 1988). These larger fruit have thicker endocarps overall that are harder to get into and thus impose higher handling costs, further reinforcing burial over immediate consumption (Vander Wall 2010). Even when the exocarp disintegrates upon burial of the seeds, the fused endocarp conveniently holds all nutlets together in a single unit. While these larger fruit may be cached initially at greater frequency, ultimately, they do result 
in lower density seed banks (Parker and Ingalls, in press ).

For small fruit, lower levels of endocarp fusion may take advantage of scatter-hoarding rodent behavior by allowing small seeds to escape immediate predation; smaller fruit do have larger seed banks (Parker and Ingalls, in press ). While small, oblate fruits may be assessed, perceived to have low handling time, and more frequently consumed immediately instead of cached, those fruits that do get cached produce a higher proportion of viable seed per fruit that break apart into many nutlets when the exocarp eventually decomposes, potentially ensuring that some nutlets may escape predation. A small, single-seeded nutlet has a better chance of escape from predation than a whole fruit. Low and medium proportions of endocarp fusion effectively result in two sizes of rewards, an initially high reward whole fruit when the rodent discovers and assesses the fruit, and a low reward after loss of soft fruit parts and the variably-sized, multiple nutlets are retrieved after burial. The overall shape of the fruit influences the degree of fruit fusion and coupled with size dictates successful dispersal.

The number of viable seeds per fruit may also act to vary the size of the perceived reward and subsequent rodent behavior. Viability of seeds is variable in Arctostaphylos (Table 8) (Keeley 1977, 1987, Parker and Kelly 1989, Kelly and Parker 1990, 1991, Peterson and Parker 2016). Because Arctostaphylos fruit have multiple seeds, the amount of reward to rodents, in the form of nutritive viable seeds, is different from fruit to fruit, even within species. The amount of reward can be masked further by variation in endocarp fusion and seed viability in species with partial fusion, and by seed viability alone in species with fully fused or free endocarps. This combination of varying viability and fusion of endocarps increases uncertainty in decision making by rodents and presumably to increase the chance that they will leave some seed in caches to build the persistent soil seed bank.

The origin of the persistent soil seed bank trait in these species occurred in the context of both fire regimes and scatter hoarding (Parker 2015b). The absence of a relationship between the proportion of fused endocarp, fruit viability, current environmental regimes, and life history suggests that these fruit traits arose early inArctostaphylos. Three other close relatives in the Arbutoideae, for example, Comarostaphylis , Ornithostaphylos, andXylococcus also have stony endocarps and in these genera all the endocarps are fused into a single structure but do not develop persistent seed banks (Parker and Stickrod, in press ). Fire regimes may have spurred the evolution of seed dormancy globally, especially in Mediterranean-climate regions (Keeley et al.2012), but of the Mediterranean-climate genera in the subfamily Arbutoideae, only Arctostaphylos developed physiological seed dormancy and persistent soil seed banks (Parker and Stickrod, in press ). Appropriate selective conditions for developing seed dormancy and obligate seed life histories were created through the combination of rodent caching and the environmental conditions produced by fire (Parker 2015b), but variation in endocarp fusion and seed viability support the persistence of the seed bank.

Fruit size and shape were principal correlates on fruit endocarp fusion and fruit viability in Arctostaphylos - Size usually sends a predictable signal to rodents about the amount of potential reward, resulting in preferential manipulation and dispersal for larger-sized fruit. This is supported in studies of oak acorns (Munoz and Bonal 2008, Zhang and Zhang 2008, Sunyer et al. 2014), palms and tropical trees (Forget et al. 1998, Brewer 2001, Jansen et al.2002, 2004), pines (Wang and Chen 2009), and mixed forests (Xiaoet al. 2006). Larger fruit size comes with increased endocarp or other tissue thickness and may also be a constraint for some animals. InLeucadendron shrubs, rodent behavior was influenced by seed size and hull thickness; medium sized seeds with medium-thick hulls were buried and small seeds with thin hulls were consumed in-situ, while large seeds were not dispersed, likely a result of being too big and thick for the resident rodents to carry (Rusch et al. 2013). Seed size plays a distinct role in the context of scatter-hoarding, in that particular rodent species will have different size preferences (Wang and Chen 2009, Wang and Yang 2020). Rodent preference for seed size or endocarp thickness may control the extent of burial versus immediate consumption (Zhang and Zhang 2008, Wang et al. 2012, Rusch et al. 2013, Zhang et al. 2016, Dylewski et al. 2020). Our results are consistent with these studies in the sense of manipulating rodent behavior. But an additional issue is that inArctostaphylos other traits also varied with fruit size and shape, specifically percent seed viability and endocarp fusion. Larger fruit tended to have lower percent viability while smaller 
fruit had lower endocarp fusion, but there was a lack of consistency in these trends. A consequence of the lack of consistency makes size alone a more ambiguous signal and underlies the patterns we observe and perhaps the success of this genus.

\section{Literature Cited}

Baker, H., 1972. Seed weight in relation to environmental conditions in California. Ecology , 53 (6), 997-1010.

Bates, D.M., Maechler, M., Bolker, B., and Walker, S., 2015. Fitting linear mixed-effects models using lme4. Journal of Statistical Software, 67, 1-48.

Bolker, B.M., Brooks, M.E., Clark, C.J., Geange, S.W., Poulsen, J.R., Stevens, M.H.H., and White, J.S.S., 2009. Generalized linear mixed models: a practical guide for ecology and evolution. Trends in Ecology and Evolution , 24 (3), 127-135.

Brewer, S.W., 2001. Predation and dispersal of large and small seeds of a tropical palm. Oikos , 92 (2), $245-255$.

Brown, J.S., 1988. Patch use as an indicator of habitat preference, predation risk, and competition. Behavioral Ecology and Sociobiology, 22, 37-47.

Burnham, K.P., Anderson, D.R., and Huyvaert, K.P., 2011. AIC model selection and multimodel inference in behavioral ecology: Some background, observations, and comparisons. Behavioral Ecology and Sociobiology , 65 (1), 23-35.

Charnov, E.L., 1976. Optimal Foraging: the Marginal Value Theorem.Theoretical population biology , 9, $129-136$.

Consortium of California Herbaria (CCH), 2018. Data provided by the participants of the Consortium of California Herbaria. [online]. Available from: www.cch2.org [Accessed 1 Oct 2018].

Dylewski, Ł., Ortega, Y.K., Bogdziewicz, M., and Pearson, D.E., 2020. Seed size predicts global effects of small mammal seed predation on plant recruitment. Ecology Letters , 23, 1024-1033.

Flint, L.E. and Flint, A.L., 2014. California Basin characterization model: a dataset of historical and future hydrologic response to climate change.

Forget, P.M., Milleron, T., and Feer, F., 1998. Patterns in post-dispersal seed removal by neotropical rodents and seed fate in relation to seed size. In : Dynamics of tropical communities . Blackwell Science Oxford, $25-49$.

Hartig, F., 2019. DHARMa: Residual diagnostics for hierarchical (Multi-Level / Mixed) regression models.

Jansen, P.A., Bartholomeus, M., Bongers, F., Elzinga, J.A., den Ouden, J., and Van Wieren, S.E., 2002. The role of seed size in dispersal by a scatter-hoarding rodent. In : D.J. Levey, W.R. Silva, and M. Galetti, eds. Seed Dispersal and Frugivory: Ecology, Evolution and Conservation. New York: CAB International, 209-225.

Jansen, P.A., Bongers, F., and Hemerik, L., 2004. Seed mass and mast seeding enhance dispersal by a neotropical scatter-hoarding rodent.Ecological Monographs , 74 (4), 569-589.

Kauffmann, M., Parker, V.T., and Vasey, M.C., 2015. Field Guide to Manzanitas . 1st ed. Kneeland: Backcountry Press.

Keeley, J.E., 1977. Seed production, seed populations in soil, and seedling production after fire for two congeneric pairs of sprouting and nonsprouting chaparral shrubs. Ecology , 58, 820-829.

Keeley, J.E., 1987. Ten years of change in seed banks of the chaparral shrubs, Arctostaphylos glauca and A. glandulosa .American Midland Naturalist, 117 (2), 446-448. 
Keeley, J.E., Bond, W.J., Bradstock, R.A., Pausas, J.G., and Rundel, P.W., 2012. Fire in Mediterranean Ecosystems: Ecology, Evolution, and Management . New York: Cambridge University Press.

Keeley, J.E. and Hays, R., 1976. Differential seed predation on two species of Arctostaphylos (Ericaceae). Oecologia , 24, 71-81.

Keeley, J.E. and Keeley, S.C., 1977. Energy allocation patterns of a sprouting and a nonsprouting species of Arctostaphylos in the California chaparral. American Midland Naturalist , 98 (1), 1-10.

Kelly, V.R. and Parker, V.T., 1990. Seed bank survival and dynamics in sprouting and nonsprouting Arctostaphylos species. American Midland Naturalist , 124 (1), 114-123.

Kelly, V.R. and Parker, V.T., 1991. Percent seed set, sprouting habit, and ploidy level in Arctostaphylos (Ericaceae). Madroño , 38 (4), 227-232.

Levine, J.M. and Murrell, D.J., 2003. The community-level consequences of seed dispersal patterns. Annual Review of Ecology, Evolution, and Systematics, 34 (November 2016), 549-574.

Lichti, N.I., Steele, M.A., and Swihart, R.K., 2015. Seed fate and decision-making processes in scatterhoarding rodents. Biological Reviews , 92 (1), 473-504.

Moles, A.T., Ackerly, D.D., Tweddle, J.C., Dickie, J.B., Smith, R., Leishman, M.R., Mayfield, M.M., Pitman, A., Wood, J.T., and Westoby, M., 2007. Global patterns in seed size. Global Ecology and Biogeography , 16 (1), 109-116.

Moore, C.M. and Vander Wall, S.B., 2015. Scatter-hoarding rodents disperse seeds to safe sites in a fire-prone ecosystem. Plant Ecology , 216 (8), 1137-1153.

Muñoz, A. and Bonal, R., 2008. Are you strong enough to carry that seed? Seed size/body size ratios influence seed choices by rodents. Animal Behaviour, 76, 709-715.

O'Neil, S.E. and Parker, V.T., 2005. Factors Contributing to the soil seed bank ize of two obligate seedling Ceanothus species in Northern California. Madroño , 52 (3), 182-190.

Parker, V.T., 2015a. Seed bank divergence between ArctostaphylosAdans. (Ericaceae) and Ceanothus L. (Rhamnaceae) suggests different seed predator interactions. Ecologia Mediterranea , 41 (2), 5-14.

Parker, V.T., 2015b. Dispersal mutualism incorporated into large-scale, infrequent disturbances. Plos One , 10 (7), e0132625.

Parker, V.T. and Ingalls, S., n.d. Seed size-seed number tradeoffs: Influence of seed size on the density of fire-stimulated persistent soil seed banks in Arctostaphylos (Ericaceae). American Journal of Botany, In press.

Parker, V.T. and Kelly, V.R., 1989. Seed banks in California chaparral and other mediterranean climate shrublands. In : M.A. Leck, V.T. Parker, and R.L. Simpson, eds. Ecology of Soil Seed Banks . New York: Academic Press, 231-255.

Parker, V.T., Vasey, M.C., and Keeley, J.E., 2009.Arctostaphylos . In : Flora of North America Editorial Committee, ed. Flora of North America North of Mexico. 20+ vols.New York and Oxford, 406-445.

Parker, V.T., Vasey, M.C., and Keeley, J.E., 2012.Arctostaphylos . In : B.G. Baldwin, D.H. Goldman, D.J. Keil, R. Patterson, T.J. Rosatti, and D.H. Wilken, eds. The Jepson Manual . Berkeley: University of California Press, 686-699.

Perea, R., López, D., San Miguel, A., and Gil, L., 2012. Incorporating insect infestation into rodent seed dispersal: Better if the larva is still inside. Oecologia , 170 (3), 723-733.

Peterson, N.B. and Parker, V.T., 2016. Dispersal by rodent caching increases seed survival in multiple ways in canopy-fire ecosystems.Ecology and Evolution , 6 (13), 4298-4306. 
PRISM Climate Group, n.d. 30-Year Normals.

R Core Team, 2018. R: A language and environment for statistical computing.

Rubio de Casas, R., Donohue, K., Venable, D.L., and Cheptou, P.O., 2015. Gene-flow through space and time: dispersal, dormancy and adaptation to changing environments. Evolutionary Ecology , 29 (6), 813-831.

Rusch, U.D., Midgley, J.J., and Anderson, B., 2013. Rodent consumption and caching behaviour selects for specific seed traits. South African Journal of Botany , 84 (January), 83-87.

Schielzeth, H., 2010. Simple means to improve the interpretability of regression coefficients. Methods in Ecology and Evolution , 1, 103-113.

Simpson, A.G., 2009. Propagule size gradients in Arctostaphylos (Ericaceae): The effects of altitude and latitude. California State University, Chico.

Simpson, A.G., Schierenbeck, K.A., Parker, V.T., and Ivey, C.T., 2017. Not all plant taxa display typical latitude-propagule size gradients : A case study In Arctostaphylos L. (Ericaceae). Madrono , 64 (2), 83-91.

Simpson, M.G., 2010. Plant Systematics. San Diego, CA: Academic Press.

Sunyer, P., Espelta, J.M., Bonal, R., and Munoz, A., 2014. Seeding phenology influences wood mouse seed choices: The overlooked role of timing in the foraging decisions by seed-dispersing rodents.Behavioral Ecology and Sociobiology , 68, 1205-1213.

Thompson, K., Band, S.R., and Hodgson, J.G., 1993. Seed size and shape predict persistence in soil. Functional Ecology , 7, 236-241.

Tiffney, B., 2004. Vertebrate dispersal of seed plants through time. Annual review of ecology, evolution, and systematics, 35 (2004), 1-29.

Venable, D.L. and Brown, J.S., 1988. The selective interactions of dispersal, dormancy, and seed size as adaptations for reducing risk in variable environments. The American Naturalist , 131 (3), 360-384.

Wahlert, G., Parker, V.T., and Vasey, M.C., 2009. A phylogeny ofArctostaphylos (Ericaceae) inferred from nuclear ribosomal ITS sequences. Journal of the Botanical Research Institute of Texas , 3 (2), 673-682.

Vander Wall, S.B., 2001. Th evolutionary ecology of nut dispersal. The Botanical Review , 67 (1), 74-117.

Vander Wall, S.B., 2010. How plants manipulate the scatter-hoarding behaviour of seed-dispersing animals. Philosophical transactions of the Royal Society Biological Sciences , 365, 989-997.

Wang, B. and Chen, J., 2009. Seed size, more than nutrient or tannin content, affects seed caching behavior of a common genus of old world rodents. Ecology , 90 (11), 3023-3032.

Wang, B. and Smith, T., 2002. Closing the seed dispersal loop.Trends in Ecology ES Evolution, 17 (8), $379-386$.

Wang, B., Wang, G., and Chen, J., 2012. Scatter-hoarding rodents use different foraging strategies for seeds from different plant species.Plant Ecology , 213 (8), 1329-1336.

Wang, B. and Yang, X., 2020. Scatter-hoarding rodent foraging preference on nutrient content is mediated by seed size. Current Zoology, 66 (4), 445-446.

Warzecha, B. and Parker, V.T., 2014. Differential post-dispersal seed predation drives chaparral seed bank dynamics. Plant Ecology , 215 (11), 1313-1322.

Xiao, Z., Wang, Y., Harris, M., and Zhang, Z., 2006. Spatial and temporal variation of seed predation and removal of sympatric large-seeded species in relation to innate seed traits in a subtropical forest, Southwest China. Forest Ecology and Management, 222 (1-3), 46-54. 
Zhang, H., Yan, C., Chang, G., and Zhang, Z., 2016. Seed trait-mediated selection by rodents affects mutualistic interactions and seedling recruitment of co-occurring tree species. Oecologia, 180, 475-484.

Zhang, H. and Zhang, Z., 2008. Endocarp thickness affects seed removal speed by small rodents in a warmtemperate broad-leafed deciduous forest, China. Acta Oecologica , 34 (3), 285-293.

Zuur, A.F., Ieno, E.N., and Elphick, C.S., 2010. A protocol for data exploration to avoid common statistical problems. Methods in Ecology and Evolution , 1 (1), 3-14.
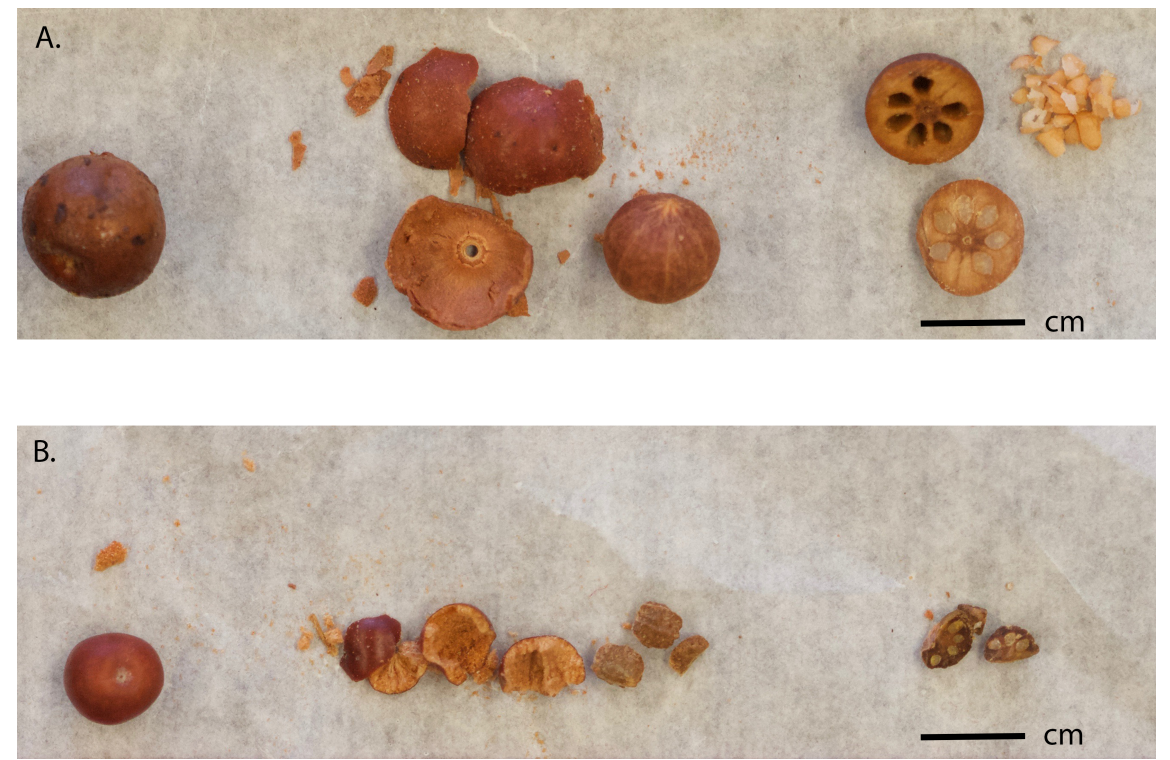

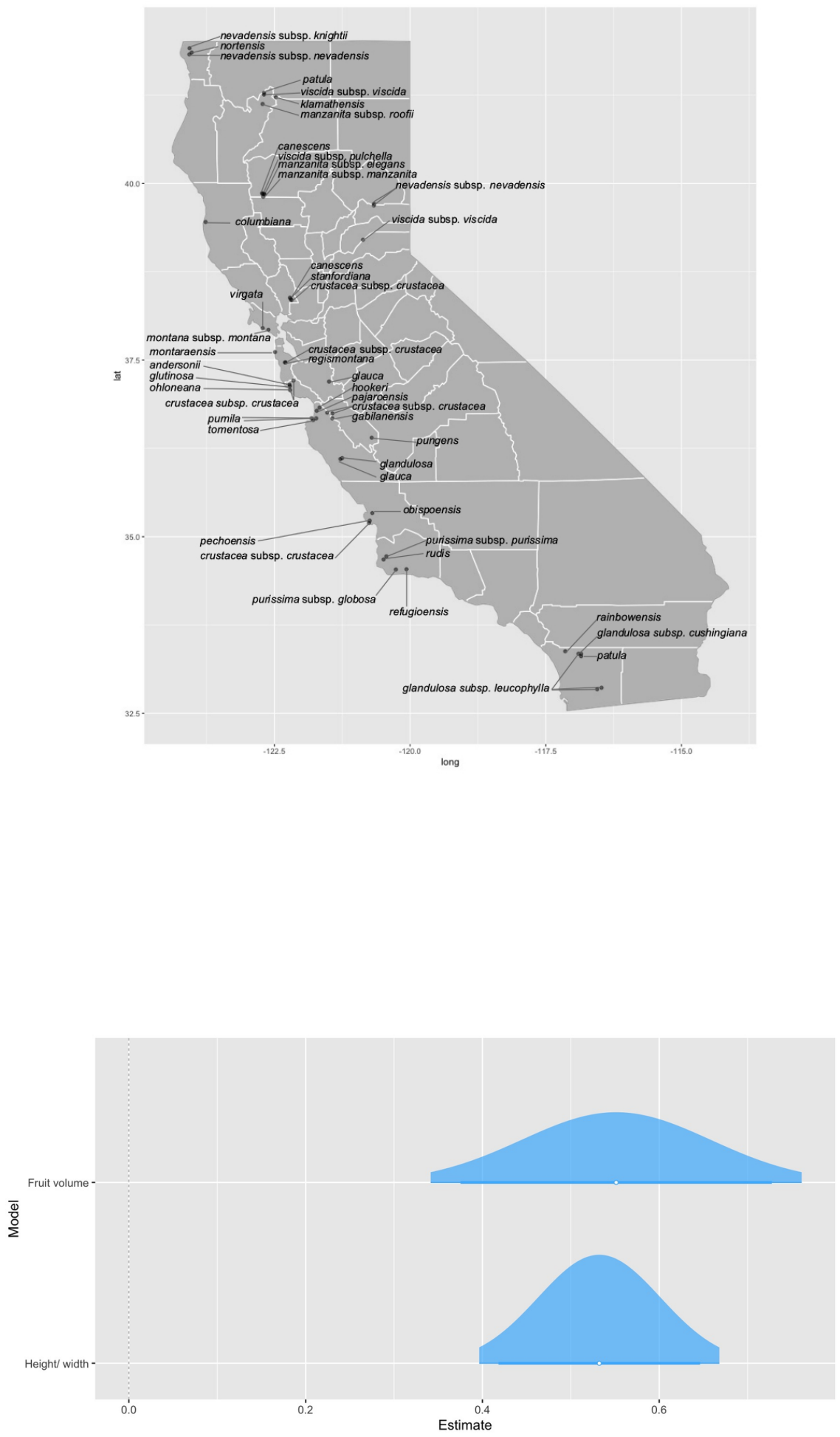

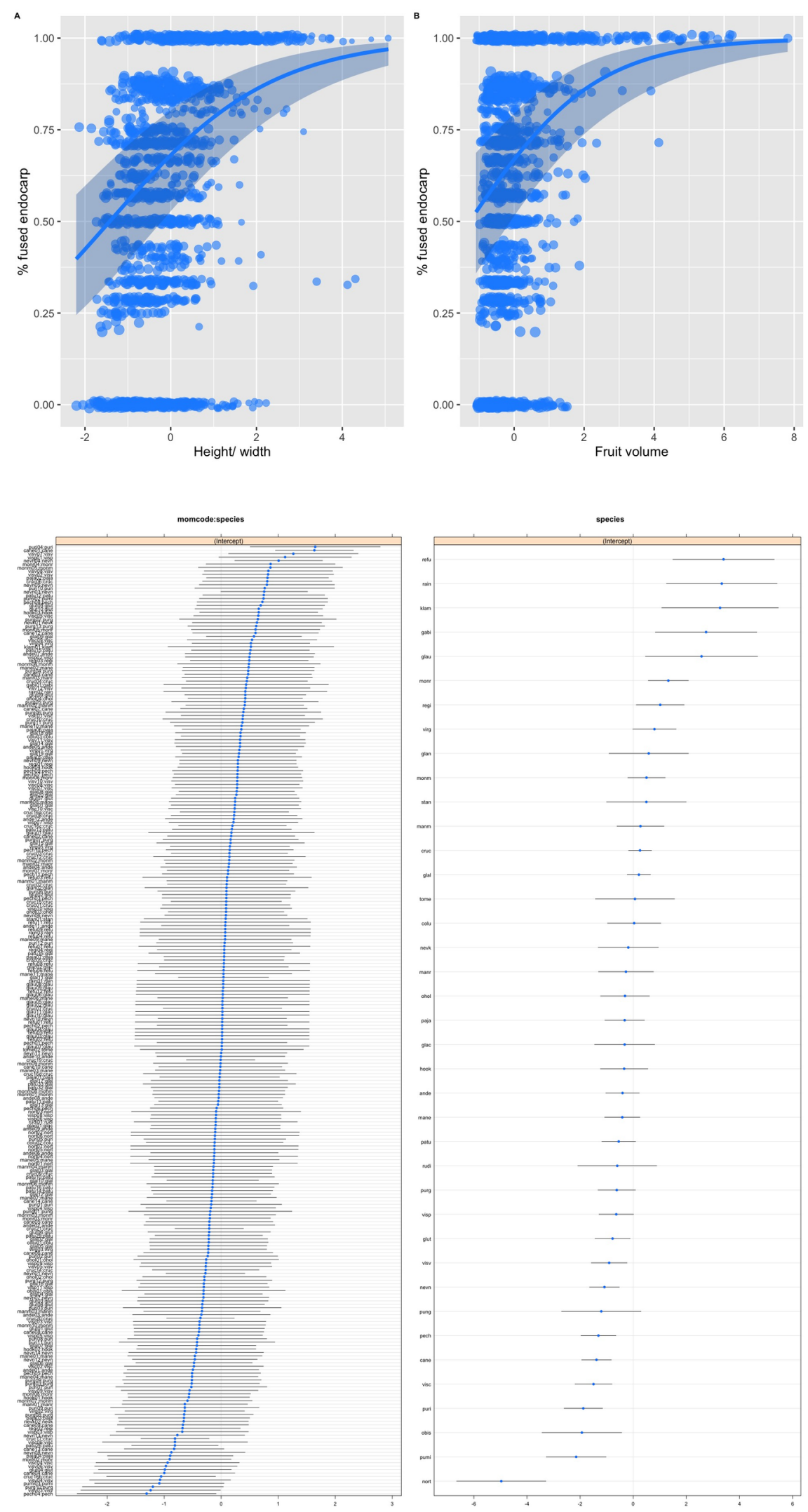

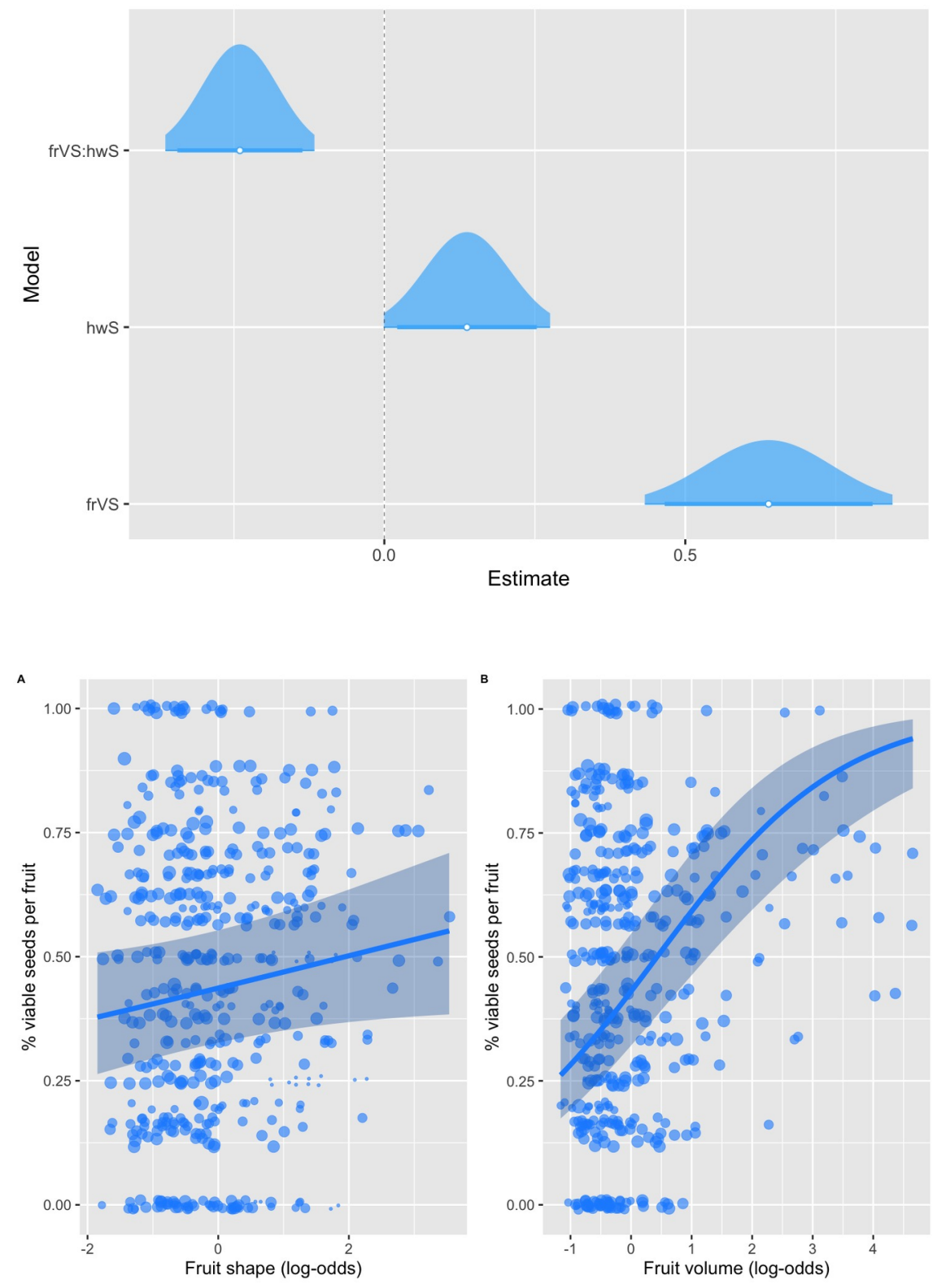


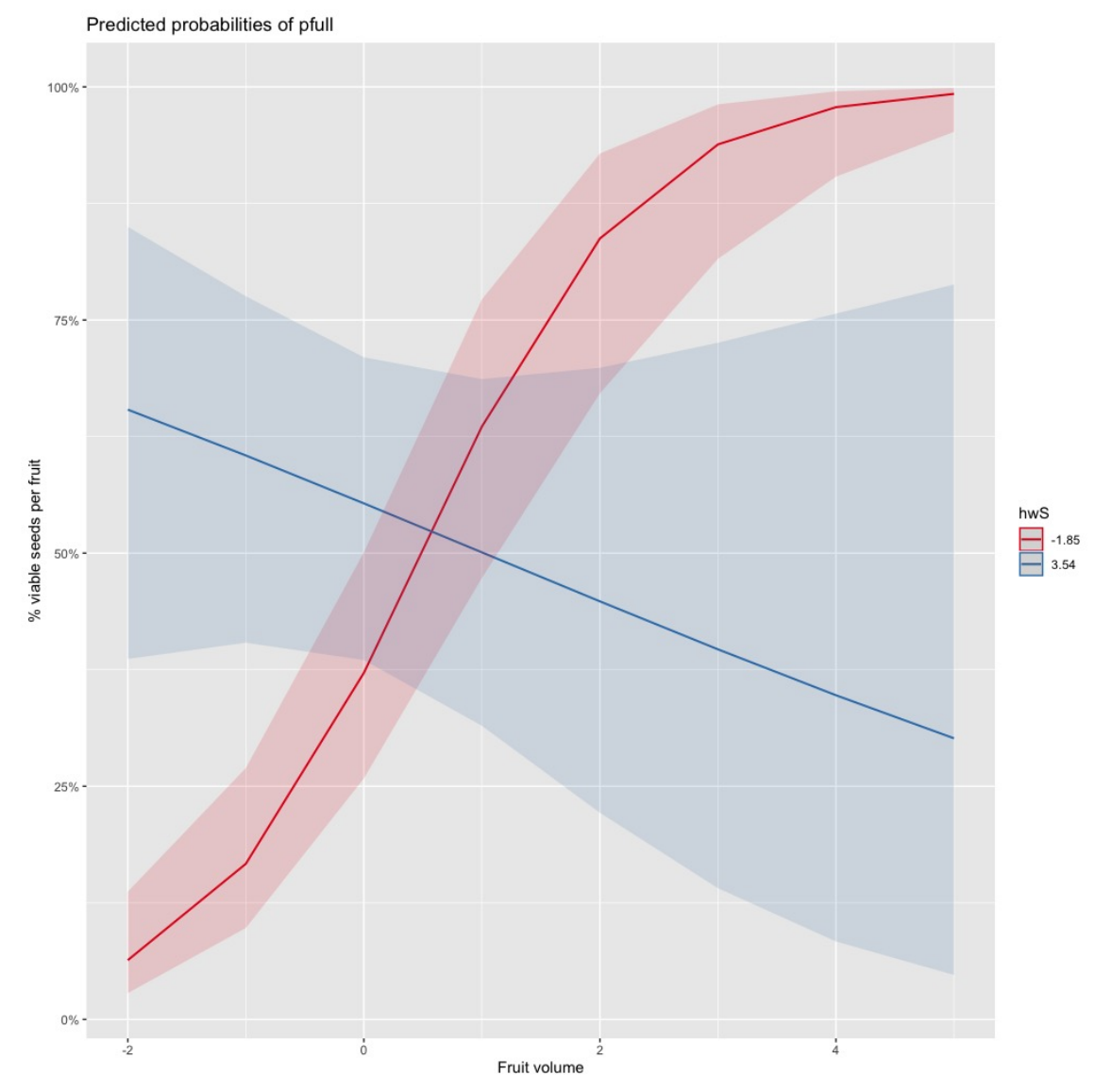



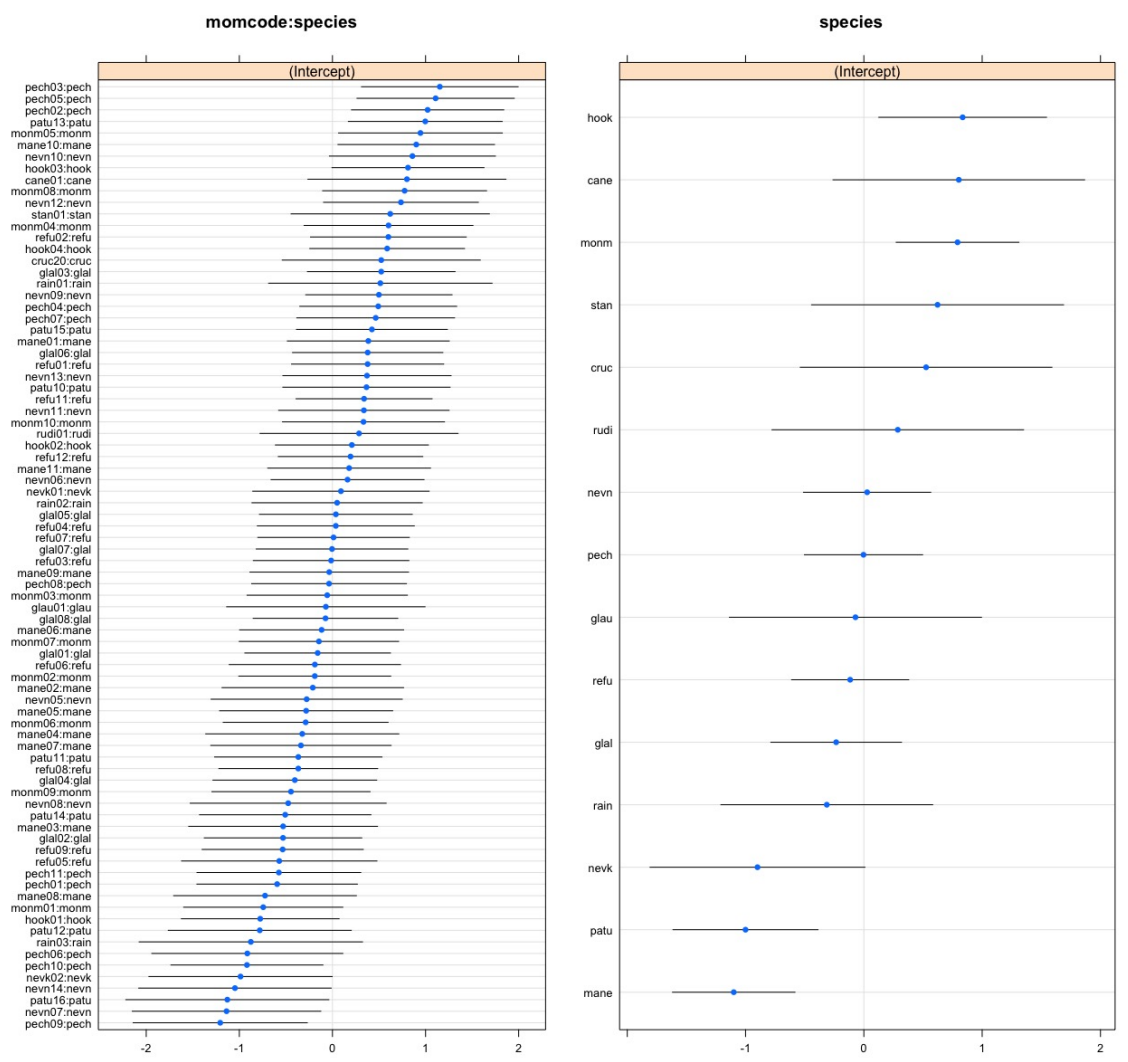